Nguyen Bac-Van (Ho Chi Minh City)

\title{
THE PRIOR DISTRIBUTION OF A RANDOM MEASURE
}

Abstract. It is known that an infinite, exchangeable sequence of observations from a Borel space, in particular a Polish one, is underlain by an almost surely (a.s.) unique random probability measure on this space such that, conditioned on it, the observations are independent and identically distributed with that measure. The distribution of that random measure is the prior distribution involved in Bayes inference. The present paper proves that the prior distribution of the a.s. unique random measure underlying an infinite, exchangeable sequence of observations from a Polish space is a Radon probability measure on the $\sigma$-field generated by the narrow topology in the space of Borel probability measures on the starting Polish space.

1. Introduction. This paper uses the notation of Kallenberg (2002, 2005).

Statistical analysis usually starts from independent and identically distributed (iid) observations whose underlying probability distribution depends on some state of nature which Bayesian statisticians consider as random. This view means postulating a random probability distribution that underlies the outcome of a statistical experiment, and the prior distribution of this random measure is acting in Bayes inference.

The present paper considers the case of an infinite, exchangeable sequence of observations from a Polish space; it is known from Theorem II.82.5 in Rogers and Williams (1994) that a space is Polish if and only if it is homeomorphic to a $G_{\delta}$ subset of $[0,1]^{\infty}$. Such an exchangeable sequence is underlain by an a.s. unique random probability measure, so the distribution of this random measure, as stated in Ferguson (1973), is the prior distribution involved in non-parametric problems.

2010 Mathematics Subject Classification: Primary 60G57; Secondary 60G09, 62A15.

Key words and phrases: conditional distribution, exchangeability, narrow topology, Radon random probability measure.

Received 9 February 2017; revised 25 June 2018.

Published online 22 February 2019. 
Now, extending a theorem of de Finetti (1931), Theorem 1.1 and Proposition 1.4(i) in Kallenberg (2005) showed that, for an infinite exchangeable sequence $\xi=\left(X_{i}, i=1,2, \ldots\right)$ of random elements $X_{i}$ in a Borel space $(S, \mathcal{S})$, there is an a.s. unique random probability measure $\nu$ on $(S, \mathcal{S})$ and a $\sigma$-field $\mathcal{F}$ in the basic probability space $(\Omega, \mathcal{A})$ such that

$$
\mathrm{P}(\xi \in \cdot \mid \mathcal{F})_{\omega}=\left(\nu_{\omega}^{\infty}\right)(\cdot) \quad \text { for almost all } \omega \in \Omega,
$$

where $\mathcal{F}$ can be replaced by $\sigma(\nu)$. In particular, for every $B \in \mathcal{S}$ and every $i$,

$$
\mathrm{P}\left(X_{i} \in B \mid \mathcal{F}\right)_{\omega}=\nu_{\omega}(B) \quad \text { for almost all } \omega \in \Omega ;
$$

in other words, $\nu$ is a conditional distribution (cd) of $X_{i}$ given $\mathcal{F}$. That is, conditioned on $\nu$, the random elements $X_{i}$ are iid with common distribution $\nu$, and the distribution of the random measure $\nu$ is just the prior distribution in Bayes inference.

Also, Kallenberg (2005, p. 25) and (2017, p. 15) stated that $\nu$ is a random element in the space $\mathcal{M}_{1}(S)$ of probability measures $\mu$ on $(S, \mathcal{S})$ endowed with the $\sigma$-field generated by all projection maps $\pi_{B}: \mu \mapsto \mu(B), B \in \mathcal{S}$. Let $g=\left(\pi_{B} ; B \in \mathcal{S}\right)$; then the map $g: \mathcal{M}_{1}(S) \rightarrow \times_{B \in \mathcal{S}} \mathbb{R}_{B}$ is a bijection onto its image. When $\mathcal{S}$ is uncountable, the Borel $\sigma$-field $\mathcal{B}\left(\mathbb{R}^{\mathcal{S}}\right)$ generated by the product topology in $\times_{B \in \mathcal{S}} \mathbb{R}_{B}$ is wider than the product $\sigma$-field $\bigotimes_{B \in \mathcal{S}} \mathcal{B}\left(\mathbb{R}_{B}\right)$, so it is desirable to extend the prior distribution of the random measure $\nu$ from the $\sigma$-field $g^{-1}\left[\bigotimes_{B \in \mathcal{S}} \mathcal{B}\left(\mathbb{R}_{B}\right)\right]$ generated by the map $g$ to $g^{-1}\left[\mathcal{B}\left(\mathbb{R}^{\mathcal{S}}\right)\right]$.

In comparison to the above situation, let $X$ be some random element $X_{i}$ in a Polish space $(S, \mathcal{S}), \mathcal{S}=\mathcal{B}(S)$; then there exists a cd $\nu$ of $X$ given a $\sigma$-field $\mathcal{F} \subset \mathcal{A}$ in $(\Omega, \mathcal{A})$. Since $S$ is Polish, $\mathcal{M}_{1}(S)$ is also the space $\mathcal{P}(S)$ of Radon probability measures on $\mathcal{B}(S)$. Therefore, from the topological viewpoint it is significant to pose the problem of extending the distribution of a cd $\nu$ of a random element in a Polish space $S$; the present paper aims at solving this problem. Such an extension for the distribution of a cd $\nu$, treated as a random measure, has not been conducted in Kallenberg (2017). We intend to prove that the prior distribution of $\nu$ is a Radon probability measure on the $\sigma$-field $\mathcal{B}(\mathcal{P}(S))$ generated by the narrow topology in the space $\mathcal{P}(S)$ of probability measures on the Polish space $S$. It is known that Radon measures permit us to avoid the image measure catastrophe; see Schwartz (1973, pp. 30-31).

The paper is organized as follows. We recall some needed facts in Section 2, with emphasis on induced measures and image measures. Our results are presented in Section 3. For any normal topological space $\mathcal{X}$, Proposition 3.1 gives a homeomorphism that transforms a random element in $\mathcal{P}(\mathcal{X})$ equipped with the narrow topology into a random element in a product space $X \mathbb{R}$ equipped with the product topology; homomorphisms into $X \mathbb{R}$ from 
$\mathcal{P}(\mathcal{X})$ are always defined through the correspondence $\mu \mapsto\left(\mu(\varphi), \varphi \in \mathcal{D}_{[a, b]}\right)$, $\mu(\varphi)=\int_{\mathcal{X}} \varphi d \mu$, where $\mathcal{D}_{[a, b]}$ is the class of all continuous $[a, b]$-valued functions with some $[a, b] \subset \mathbb{R}$. Then Corollary $3.1(1)$ of Proposition 3.4 states that the conditional distribution $\nu$ of a random element in a Polish space $S$ can be viewed as a random point in a product space $X \mathbb{R}$ which has a distribution on the product $\sigma$-field $\otimes \mathcal{B}$, the homeomorphic image of which is contained in the Borel $\sigma$-field $\mathcal{B}(\mathcal{P}(S))$ of $\mathcal{P}(S)$ endowed with the narrow topology. In order to use Corollary 3.1.(1), Proposition 3.3 offers a homeomorphism $\theta$ from a Polish space into a compact Hausdorff space $\Sigma$; by $\theta$, the conditional distribution $\nu$ in Corollary 3.1(1) is transformed into a random point $\hat{\nu}$ in the space $\mathcal{P}(\Sigma)$ of Radon probabilities on $\Sigma$, and $\mathcal{P}(\Sigma)$ is compact by Proposition 3.2 . Then, using the set $\Psi$ of all continuous $[0,1]$-valued functions $\psi$ on $\Sigma$ as the index set, we shall extend a distribution on the product $\sigma$-field $\bigotimes_{\psi \in \Psi} \mathcal{B}_{\psi}$, the homeomorphic image of which is contained in $\mathcal{B}(\mathcal{P}(\Sigma)$ ). In addition, from the random point $\hat{\nu}$ in $\mathcal{P}(\Sigma)$, the family $\hat{\zeta}(\hat{\nu})=(\hat{\nu}(\psi), \psi \in \Psi), \hat{\nu}(\psi)=\int_{\Sigma} \psi d \hat{\nu}$, generates a consistent system of finite-dimensional probability distributions $\left(\mu_{F}, F\right.$ finite $\left.\subset \Psi\right)$ which determines the Kolmogorov distribution $\mu^{\prime}$ of the process $\hat{\zeta}(\hat{\nu})=(\hat{\nu}(\psi), \psi \in \Psi)$. Then we show that the measure induced by $\mu^{\prime}$ is the distribution of $\hat{\zeta}(\hat{\nu})$ on the Borel $\sigma$-field over the relative product topology in the range space of $\hat{\zeta}(\hat{\nu})$; this is the result of Proposition 3.5, which leads to the distribution of $\hat{\nu}$ on a Borel subset of $\mathcal{P}(\Sigma)$. Finally, using the inverse of the homeomorphism from $\mathcal{P}(S)$ into $\mathcal{P}(\Sigma)$, we get the distribution of the random measure $\nu$ on $\mathcal{B}(\mathcal{P}(S))$, attaining the aim of the paper in Theorem 3.1.

\section{Preliminaries}

\subsection{Radon probabilities}

Definition 2.1. A Radon probability measure $\mu$ on a Hausdorff topological space $\mathcal{X}$ is a Borel measure, i.e. the domain of $\mu$ is the Borel $\sigma$-field $\mathcal{B}(\mathcal{X})$, which is inner regular, i.e.

$$
(\forall B \in \mathcal{B}(\mathcal{X})) \quad \mu(B)=\sup \{\mu(K): K \text { compact, } K \subset B\} .
$$

The space of Radon probability measures on $\mathcal{X}$ will be denoted by $\mathcal{P}(\mathcal{X})$.

Below, $\widehat{\mathcal{B}(\mathcal{X})_{\mu}}$ denotes the $\mu$-completion of $\mathcal{B}(\mathcal{X})$; see Kallenberg 2002

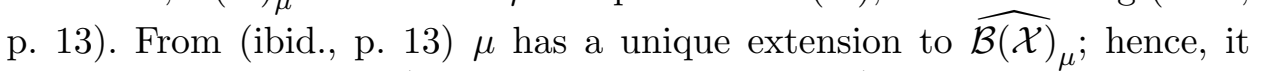
follows from Schwartz $(1973$, Proposition 1, p. 18) that the above inner regularity holds on $\widehat{\mathcal{B}(\mathcal{X})_{\mu}}$.

From Schwartz (1973, Definition $R_{2}$, p. 13), a Radon probability $\mu$ is outer regular, that is,

$$
\left(\forall B \in \widehat{\mathcal{B}(\mathcal{X})_{\mu}}\right) \quad \mu(B)=\inf _{O \text { open } \supset B} \mu(O) .
$$


From the inner and outer regularity of $\mu$, it follows that a Radon probability measure $\mu$ is a regular set function (cf. Dunford and Schwartz 1958, Definition 11, p. 137)) on $\widehat{\mathcal{B}(\mathcal{X})}{ }_{\mu}$.

If every Borel probability measure on a Hausdorff topological space $\mathcal{X}$ is a Radon probability, $\mathcal{X}$ is called a Radon space. From Oxtoby and Ulam (1939), every Polish space is Radon.

\subsection{Induced measures}

Definition 2.2. Let $(\mathcal{X}, \mathcal{E}, m)$ be a measure space and $Y \subset \mathcal{X}$. Put $\mathcal{E}_{Y}=\mathcal{E} \cap Y=\{A \cap Y: A \in \mathcal{E}\}$; then $Y \in \mathcal{E}_{Y}$. The set function $m_{Y}$ defined on the $\sigma$-field $\mathcal{E}_{Y}$ by $m_{Y}(B)=\inf \{m(A): A \in \mathcal{E}, A \supset B\}$ is called the measure induced by $m$ on the subset $Y$.

From Schwartz (1973, p. 20), it is known that $m_{Y}$ is countably additive, so $m_{Y}$ is a measure on $\mathcal{E}_{Y}$.

\subsection{Image measures}

Definition 2.3. Let $(\mathcal{X}, \mathcal{E}, m)$ be a measure space and $(\Gamma, \mathcal{C})$ a measurable space. Let $g:(\mathcal{X}, \mathcal{E}) \rightarrow(\Gamma, \mathcal{C})$ be an $m$-measurable mapping, i.e. $g^{-1}(\mathcal{C}) \subset \hat{\mathcal{E}}_{m}$, the $m$-completion of $\mathcal{E}$. Then, by using the unique extension of $m$ to $\hat{\mathcal{E}}_{m}$, the measure, denoted by $g m$, defined on $\mathcal{C}$ by

$$
(g m)(C)=m\left(g^{-1}(C)\right) \quad \forall C \in \mathcal{C},
$$

is called the image measure of $m$ by the map $g$.

Proposition 2.1. Let $\mathcal{X}, \mathcal{Y}$ be Hausdorff topological spaces, $\mu$ a Radon probability on $\mathcal{X}$, and $g: \mathcal{X} \rightarrow \mathcal{Y}$ a continuous map. Then the image measure $g \mu$ is a Radon probability on $\mathcal{Y}$.

See Schwartz (1973, pp. 36-37).

2.4. Narrow topology. In Schwartz (1973, p. 370, Definition 1), the narrow topology was defined in the space of finite Radon measures on an arbitrary Hausdorff topological space, and in Proposition 2, p. 371 there, it was proved that this topology is Hausdorff. We shall only use the following narrow topology definition in Schwartz (1973, Part II, Chap. V, §1) for a completely regular space:

$\mathcal{X}$ being a completely regular space, a generalized sequence $\left\{\mu_{d}\right\}$ in $\mathcal{P}(\mathcal{X})$ is said to converge narrowly to $\mu \in \mathcal{P}(\mathcal{X})$ if, $\mathcal{D}$ denoting the collection of all continuous $[0,1]$-valued functions $\varphi$ on $\mathcal{X}$,

$$
(\forall \varphi \in \mathcal{D}) \quad \lim _{d} \mu_{d}(\varphi)=\mu(\varphi), \quad \mu(\varphi)=\int_{\mathcal{X}} \varphi d \mu .
$$

Hence, from (ibid., Theorem 7, p. 385) we obtain 
Proposition 2.2. If $\mathcal{X}$ is a Polish space, $\mathcal{P}(\mathcal{X})$ equipped with the narrow topology is Polish.

Furthermore, we get

Proposition 2.3. Let $g$ be a homeomorphism from a Hausdorff space $\mathcal{X}$ onto a Hausdorff space $\mathcal{Z}$, and $\mathcal{P}(\mathcal{X}), \mathcal{P}(\mathcal{Z})$ be equipped with the narrow topologies. Then the map $h: \mu \mapsto g \mu$ is a homeomorphism from $\mathcal{P}(\mathcal{X})$ onto $\mathcal{P}(\mathcal{Z})$.

Proof. By assumption, $g: \mathcal{X} \rightarrow \mathcal{Z}$ and $g^{-1}: \mathcal{Z} \rightarrow \mathcal{X}$ are continuous. Thus, the conditions of Theorem 11 in Schwartz (1973, p. 37) are fulfilled; therefore, every $\nu \in \mathcal{P}(\mathcal{Z})$ is the image $g \mu$ of some $\mu \in \mathcal{P}(\mathcal{X})$. From (ibid., Theorem 10, p. 37), the map $h: \mu \mapsto g \mu$ on $\mathcal{P}(\mathcal{X})$ to $\mathcal{P}(\mathcal{Z})$ is injective, so it is a bijection from $\mathcal{P}(\mathcal{X})$ onto $\mathcal{P}(\mathcal{Z})$. From Schwartz (1973, Proposition 3, p. 372), with $\mathcal{P}(\mathcal{X})$ and $\mathcal{P}(\mathcal{Z})$ equipped with the narrow topologies the bijection $h$ is continuous, and so is the inverse bijection $h^{-1}: \nu \mapsto g^{-1} \nu$ from $\mathcal{P}(\mathcal{Z})$ onto $\mathcal{P}(\mathcal{X})$. Thus, $h$ is a homeomorphism from $\mathcal{P}(\mathcal{X})$ onto $\mathcal{P}(\mathcal{Z})$.

\section{Conditional probability distributions}

3.1. Narrow topology: the case of normal topological spaces. In the space rca $(\mathcal{X}, \mathcal{B}(\mathcal{X}))$ (cf. Dunford and Schwartz, 1958, pp. 161-162) of all regular countably additive real-valued set functions defined on the Borel $\sigma$-field of a topological space $\mathcal{X}$, we shall also introduce the narrow topology.

Definition 3.1. For $\mathcal{X}$ completely regular, a generalized sequence $\left\{\mu_{d}\right\}$ in $\operatorname{rca}(\mathcal{X}, \mathcal{B}(\mathcal{X}))$ is said to converge narrowly to $\mu \in \operatorname{rca}(\mathcal{X}, \mathcal{B}(\mathcal{X}))$ if

$$
\lim _{d} \mu_{d}(\varphi)=\mu(\varphi) \quad \forall \varphi \in \mathcal{D} .
$$

By the regularity of Radon probability measures, always $\mathcal{P}(\mathcal{X}) \subset$ $\operatorname{rca}(\mathcal{X}, \mathcal{B}(\mathcal{X}))$

First, we get

Proposition 3.1. The narrow topology in $\operatorname{rca}(\mathcal{X}, \mathcal{B}(\mathcal{X}))$ is Hausdorff for a normal topological space $\mathcal{X}$, and on $\operatorname{rca}(\mathcal{X}, \mathcal{B}(\mathcal{X}))$ equipped with the narrow topology the map $\zeta: \mu \mapsto(\mu(\varphi), \varphi \in \mathcal{D})$ is a homeomorphism into $\times_{\varphi \in \mathcal{D}} \mathbb{R}_{\varphi}$ equipped with the product topology. In particular, $\zeta$ is a homeomorphism from $\mathcal{P}(\mathcal{X})$ equipped with the relative narrow topology onto the subspace $\zeta(\mathcal{P}(\mathcal{X}))$ of $\mathrm{X}_{\varphi \in \mathcal{D}} \mathbb{R}_{\varphi}$ equipped with the relative product topology.

Proof. Let $p \in \operatorname{rca}(\mathcal{X}, \mathcal{B}(\mathcal{X}))$ be such that $p(\varphi)=\int_{\mathcal{X}} \varphi d p \geq 0$ for all $\varphi \in \mathcal{D}$. Let us show that $p$ is non-negative.

By Hahn's decomposition (cf. Dunford and Schwartz, 1958, Theorem III.4.10, p. 129), there is a set $E_{0} \in \mathcal{B}(\mathcal{X})$ such that $p$ is non-negative on 
subsets of $E_{0}$ and non-positive on subsets of $E_{0}^{c}$. By the definition of regular set functions (cf. ibid., p. 137),

$(\forall \epsilon>0)\left(\exists\right.$ a closed set $\left.F \subset E_{0}\right)\left(\exists\right.$ an open set $\left.G \supset E_{0}\right)$

$$
(\forall C \in \mathcal{B}(\mathcal{X}), C \subseteq G-F) \quad|p(C)|<\epsilon .
$$

Then for each $n$, there are a closed set $F_{n} \subset E_{0}$ and an open set $G_{n} \supset E_{0}$ such that $|p(C)|<1 / n$ for all $C \subseteq G_{n}-F_{n}$; moreover, $G_{n+1}$ can be replaced by $G_{n+1}^{\prime}=G_{n} \cap G_{n+1}$, so we can assume that the sequence $\left\{G_{n}\right\}$ is nonincreasing.

Suppose that $p\left(G_{n_{0}}^{c}\right)<0$ for some $n_{0}$. In Dunford and Schwartz 1958, p. 127), it was proved that a real-valued countably additive set function defined on a $\sigma$-field in an arbitrary set is always bounded, so the sequence $\left\{-p\left(G_{n}^{c}\right)\right\}$ is bounded and non-decreasing, hence converges to a positive limit $\beta$, say. From Urysohn's theorem, there is $f \in \mathcal{D}$ such that $f(F)=0$ and $f\left(G^{c}\right)=1$; in addition,

$$
\left|\int_{G-F} f d p\right| \leq p\left(E_{0}-F\right)+(-p)\left(G-E_{0}\right) .
$$

Thus, $\left|\int_{G-F} f d p\right| \leq 2 \epsilon$. In particular, if $f_{n} \in \mathcal{D}$ is such that $f_{n}\left(F_{n}\right)=0$ and $f_{n}\left(G_{n}^{c}\right)=1$, then $\left|\int_{G_{n}-F_{n}} f_{n} d p\right| \leq 2 / n$. Furthermore, for $p(f)=\int_{\mathcal{X}} f d p$ $\geq 0$, by assumption we have

$$
p(f)=\int_{G^{c}} f d p+\int_{G-F} f d p=p\left(G^{c}\right)+\int_{G-F} f d p ;
$$

in particular,

$$
0 \leq p\left(f_{n}\right)=p\left(G_{n}^{c}\right)+\int_{G_{n}-F_{n}} f_{n} d p,
$$

so $\lim _{n \rightarrow \infty} p\left(f_{n}\right)=-\beta<0$, which is a contradiction.

Therefore, $p\left(G_{n}^{c}\right)=0$ for all $n$ because $p\left(G_{n}^{c}\right) \leq 0$; moreover,

$$
p\left(E_{0}^{c}\right)=p\left(G_{n}^{c}\right)+p\left(G_{n}-E_{0}\right)=p\left(G_{n}-E_{0}\right),
$$

so $\left|p\left(E_{0}^{c}\right)\right|=\left|p\left(G_{n}-E_{0}\right)\right|<1 / n$ for all $n$. Thus, $p\left(E_{0}^{c}\right)=0$, so $p$ is nonnegative on $\mathcal{B}(\mathcal{X})$.

If a generalized sequence $\left\{\mu_{d}\right\}$ in $\operatorname{rca}(\mathcal{X}, \mathcal{B}(\mathcal{X}))$ has two limits $\mu$ and $\mu^{\prime}$ in the narrow topology then $\left(\mu-\mu^{\prime}\right)(\varphi)=\left(\mu^{\prime}-\mu\right)(\varphi)=0$ for all $\varphi \in \mathcal{D}$, so $\mu-\mu^{\prime}$ and $\mu^{\prime}-\mu$ are non-negative. Thus, $\mu=\mu^{\prime}$, i.e. the narrow topology in $\operatorname{rca}(\mathcal{X}, \mathcal{B}(\mathcal{X}))$ is Hausdorff.

Let $\mu, \mu^{\prime} \in \operatorname{rca}(\mathcal{X}, \mathcal{B}(\mathcal{X}))$. Then $\zeta(\mu)=\zeta\left(\mu^{\prime}\right)$ means $\mu(\varphi)=\mu^{\prime}(\varphi)$, or $\left(\mu-\mu^{\prime}\right)(\varphi)=0$, for all $\varphi \in \mathcal{D}$; thus, $\mu=\mu^{\prime}$. Hence, $\zeta$ is an injection from $\operatorname{rca}(\mathcal{X}, \mathcal{B}(\mathcal{X}))$ into $\times_{\varphi \in \mathcal{D}} \mathbb{R}_{\varphi}$. Since $\zeta(\operatorname{rca}(\mathcal{X}, \mathcal{B}(\mathcal{X}))) \subset \times_{\varphi \in \mathcal{D}} \mathbb{R}_{\varphi}$, to the relative product topology of $\zeta(\operatorname{rca}(\mathcal{X}, \mathcal{B}(\mathcal{X})))$ there corresponds by the bijection $\zeta^{-1}$ a topology $\tau_{P}$ in $\operatorname{rca}(\mathcal{X}, \mathcal{B}(\mathcal{X}))$; then $\zeta$ is a homeomorphism 
from $\left(\operatorname{rca}(\mathcal{X}, \mathcal{B}(\mathcal{X})), \tau_{P}\right)$ onto $\zeta(\operatorname{rca}(\mathcal{X}, \mathcal{B}(\mathcal{X})))$ equipped with the relative product topology. Moreover, $\tau_{P}$ is just the narrow topology since the $\tau_{P^{-}}$ convergence of a generalized sequence $\left\{\mu_{d}\right\}$ to $\mu$ in $\operatorname{rca}(\mathcal{X}, \mathcal{B}(\mathcal{X}))$ means $\zeta\left(\mu_{d}\right) \rightarrow \zeta(\mu)$ in the product space $\times_{\varphi \in \mathcal{D}} \mathbb{R}_{\varphi}$, or $\mu_{d}(\varphi) \rightarrow \mu(\varphi)$ for all $\varphi \in \mathcal{D}$.

Propositions 3.2 below will serve the proof of Proposition 3.5 .

Proposition 3.2. If $\mathcal{X}$ is a compact Hausdorff space, then the set $\mathcal{P}(\mathcal{X})$ of all Radon probabilities on $\mathcal{B}(\mathcal{X})$ is also a compact Hausdorff space in the narrow topology.

Proof. The space $\mathcal{X}$ being compact, by the F. Riesz representation theorem in Dunford and Schwartz (1958, p. 265), $\mathrm{C}(\mathcal{X})$ being the space of all bounded continuous functions on $\mathcal{X}$, each element $\mu$ of the B-space $\operatorname{rca}(\mathcal{X}, \mathcal{B}(\mathcal{X}))$ can be identified with an element $z \in \mathrm{C}^{*}(\mathcal{X})$ such that $z \varphi=\mu(\varphi)$ for all $\varphi \in \mathrm{C}(\mathcal{X})$. Then a generalized sequence $\left\{z_{d}\right\}$ in $\mathrm{C}^{*}(\mathcal{X})$ converges to $z \in \mathrm{C}^{*}(\mathcal{X})$ in the weak* topology of $\mathrm{C}^{*}(\mathcal{X})$ (cf. Dunford and Schwartz, 1958, p. 420) if and only if the corresponding $\left\{\mu_{d}, d \in D\right\}$ converges to $\mu$ in the narrow topology of $\operatorname{rca}(\mathcal{X}, \mathcal{B}(\mathcal{X}))$. Thus, to the narrowly closed set $\mathcal{P}(\mathcal{X})$ in $\operatorname{rca}(\mathcal{X}, \mathcal{B}(\mathcal{X}))$ there corresponds a weak ${ }^{*}$-closed set $F$ in $\mathrm{C}^{*}(\mathcal{X})$, and, since $\mathcal{P}(\mathcal{X})$ is bounded in the $\mathrm{B}$-space $\operatorname{rca}(\mathcal{X}, \mathcal{B}(\mathcal{X}))$, by isometric correspondence $F$ is bounded in the norm topology of the B-space $\mathrm{C}^{*}(\mathcal{X})$; according to Dunford and Schwartz (1958, Corollary V.4.3, p. 424), $F$ is compact in the weak* topology of $\mathrm{C}^{*}(\mathcal{X})$ since it is closed in the weak* topology and bounded in the norm topology. By the homeomorphism between $\operatorname{rca}(\mathcal{X}, \mathcal{B}(\mathcal{X}))$ equipped with the narrow topology and $\mathrm{C}^{*}(\mathcal{X})$ equipped with the weak* topology, the set $\mathcal{P}(\mathcal{X})$ corresponding to the compact set $F$ is compact in the narrow topology of $\operatorname{rca}(\mathcal{X}, \mathcal{B}(\mathcal{X}))$ which is Hausdorff by Proposition 3.1 thus, $\mathcal{P}(\mathcal{X})$ is compact Hausdorff.

The following proposition, which appeared in a lecture given by Laurent Schwartz in Hanoi, April 1976, specifies Theorem 22 in Dunford and Schwartz (1958, p. 276).

Proposition 3.3. Let $S$ be a completely regular topological space; then

- the map $\theta: S \ni s \mapsto(\varphi(s), \varphi \in \mathcal{D}) \in[0,1]^{\mathcal{D}}$ is a homeomorphism from $S$ onto $\theta(S)$ supplied with the relative topology from the product topology in $[0,1]^{\mathcal{D}}$,

- the closure $\Sigma=\overline{\theta(S)}$ is a compact Hausdorff space.

Proof. The map $\theta$ is injective, for given $s, s^{\prime} \in S, s \neq s^{\prime}$, by the complete regularity there is a $\varphi \in \mathcal{D}$ such that $\varphi(s) \neq \varphi\left(s^{\prime}\right)$; it is continuous, for given any generalized sequence $s_{d}$ converging to $s, \theta\left(s_{d}\right)$ converging to $\theta(s)$ according to the product topology is equivalent to the convergence of every 
coordinate $\theta_{\varphi}\left(s_{d}\right)=\varphi\left(s_{d}\right)$ to $\theta_{\varphi}(s)=\varphi(s)$. To show that the map $\theta$ is open, let $U$ be an open set in $S$. Fix a point $s \in U$; then there exists $\varphi_{0} \in \mathcal{D}$ with $\varphi_{0}(s)=1$ and $\varphi_{0}\left(U^{c}\right)=0$, so if $\varphi_{0}\left(s^{\prime}\right) \neq 0$ then $s^{\prime} \in U$. Consider

$$
A=\left\{z: z=\left(z_{\varphi}, \varphi \in \mathcal{D}\right) \in[0,1]^{\mathcal{D}}, z_{\varphi_{0}} \in(0,1]\right\} \subset[0,1]^{\mathcal{D}} ;
$$

then $A$ is an open cylinder in $[0,1]^{\mathcal{D}}$ equipped with the product topology, so, in the subspace $\theta(S), A \cap \theta(S)$ is open. In addition,

$$
A \cap \theta(S)=\left\{z: z=\left(\varphi\left(s^{\prime}\right), \varphi \in \mathcal{D}\right), s^{\prime} \in S, \varphi_{0}\left(s^{\prime}\right) \neq 0\right\},
$$

so $A \cap \theta(S) \subset\left\{z: z=\left(\varphi\left(s^{\prime}\right), \varphi \in \mathcal{D}\right), s^{\prime} \in U\right\}=\theta(U)$. Now, $s$ being an arbitrary point in $U, \theta(s)$ is any point in $\theta(U)$, and $\theta(s) \in A \cap \theta(S)$ since the coordinate $(\theta(s))_{\varphi_{0}}=\varphi_{0}(s)$ equals $1 \in(0,1]$; moreover, $A \cap \theta(S)$ is a neighborhood of $\theta(s)$, so any point in $\theta(U)$ has a neighborhood contained in $\theta(U)$. Hence, in the relative product topology of $\theta(S), \theta(U)$ is an open set, so the map $\theta$ is open; therefore $\theta$ is a homeomorphism from $S$ onto $\theta(S) \subset[0,1]^{\mathcal{D}}$.

By the Tikhonov theorem, $[0,1]^{\mathcal{D}}$ is a compact Hausdorff space, so $\Sigma=$ $\overline{\theta(S)}$ is compact Hausdorff.

\subsection{Conditional probability distributions as Radon random} measures. We start from a random element $X$ mapping the basic probability space $(\Omega, \mathcal{A}, \mathrm{P})$ into a Polish space $S$ supplied with its Borel $\sigma$-field $\mathcal{B}(S)$. Since every Polish space is a Borel space, from Kallenberg (2002, Theorem 6.3 , p. 107) there always exists a conditional distribution $\nu$ of $X$ given a sub- $\sigma$-field $\mathcal{F} \subset \mathcal{A}$. The following proposition leads to Corollary 3.1 as mentioned in the Introduction.

Proposition 3.4. Let $\mathcal{X}$ be a normal topological space, and $\bar{\eta}: \Omega \rightarrow$ $\mathcal{P}(\mathcal{X})$ be an $\mathcal{F}$-measurable Radon probability measure on $\mathcal{B}(\mathcal{X})$; that is, $\bar{\eta}_{\omega}$ is a Radon probability measure for each $\omega \in \Omega$, and for every $B \in \mathcal{B}(\mathcal{X})$, $\bar{\eta}_{\omega}(B)$ is $\mathcal{F}$-measurable in $\omega$. Let $\zeta(\mu)=(\mu(\varphi), \varphi \in \mathcal{D})$ for $\mu \in \mathcal{P}(\mathcal{X})$. Then, the space $\mathcal{P}(\mathcal{X})$ being equipped with the narrow topology, for $\mathcal{B}_{\varphi}=\mathcal{B}\left(\mathbb{R}_{\varphi}\right)$, $\bar{\eta}$ has a probability distribution on the $\sigma$-field $\zeta^{-1}\left(\bigotimes_{\varphi \in \mathcal{D}} \mathcal{B}_{\varphi}\right)$ contained in the Borel $\sigma$-field $\mathcal{B}(\mathcal{P}(\mathcal{X}))$.

Proof. Since $\bar{\eta}(\varphi)=\int_{\mathcal{X}} \varphi d \bar{\eta}$ is $\mathcal{F}$-measurable in $\omega$ for any $\varphi \in \mathcal{D}, \zeta(\bar{\eta})=$ $(\bar{\eta}(\varphi), \varphi \in \mathcal{D})$ is a family of $\mathcal{F}$-measurable random variables. Therefore, the $\mathcal{F}$-measurable random process $\zeta(\bar{\eta})$ has a probability distribution on the product $\sigma$-field $\bigotimes_{\varphi \in \mathcal{D}} \mathcal{B}_{\varphi}$. Moreover, from Proposition 3.1, $\zeta$ is a homeomorphism from $\mathcal{P}(\mathcal{X})$ equipped with the relative narrow topology into the product space $X_{\varphi \in \mathcal{D}} \mathbb{R}_{\varphi}$; thus, $\bar{\eta}$ has a probability distribution on the $\sigma$-field $\zeta^{-1}\left(\bigotimes_{\varphi \in \mathcal{D}} \mathcal{B}_{\varphi}\right)$, which is contained in the $\sigma$-field $\mathcal{B}(\mathcal{P}(\mathcal{X}))$ generated by the narrow topology since $\bigotimes_{\varphi \in \mathcal{D}} \mathcal{B}_{\varphi}$ is contained in the Borel $\sigma$-field 
generated by the product topology in $\times_{\varphi \in \mathcal{D}} \mathbb{R}_{\varphi}$, whose inverse image by the homeomorphism $\zeta$ is $\mathcal{B}(\mathcal{P}(\mathcal{X}))$.

Corollary 3.1. $S$ being a Polish space, let $\zeta(\mu)=(\mu(\varphi), \varphi \in \mathcal{D})$ for $\mu \in \mathcal{P}(S)$.

(1) Let $\nu$ be a cd given $\mathcal{F}$ of a random element $X$ in $S$. Then $\nu$ has a probability distribution on the $\sigma$-field $\zeta^{-1}\left(\bigotimes_{\varphi \in \mathcal{D}} \mathcal{B}_{\varphi}\right)$ contained in the Borel $\sigma$-field $\mathcal{B}(\mathcal{P}(S))$ of the space $\mathcal{P}(S)$ equipped with the narrow topology.

(2) Furthermore, the image measure $\theta \nu$ by the map $\theta: S \rightarrow \Sigma$ in Proposition 3.3 is a cd given $\mathcal{F}$ of $\theta \circ X$; then, with $\hat{\nu}$ denoting $\theta \nu, \hat{\nu}(\omega) \in \mathcal{P}(\Sigma)$ for each $\omega \in \Omega$.

(3) Let $\Psi$ be the set of continuous $[0,1]$-valued functions on $\Sigma$, and $\mathcal{B}_{\psi}=$ $\mathcal{B}\left(\mathbb{R}_{\psi}\right)$ for $\psi \in \Psi$. For $\lambda \in \mathcal{P}(\Sigma)$, let $\hat{\zeta}(\lambda)=(\lambda(\psi), \psi \in \Psi)$ where $\lambda(\psi)=\int_{\Sigma} \psi d \lambda$. Then $\hat{\nu}$ has a probability distribution on the $\sigma$-field $\hat{\zeta}^{-1}\left(\bigotimes_{\psi \in \Psi} \mathcal{B}_{\psi}\right)$ contained in $\mathcal{B}(\mathcal{P}(\Sigma))$, the Borel $\sigma$-field of $\mathcal{P}(\Sigma)$ equipped with the narrow topology.

Later, Theorem 3.1 will show that in (1), $\nu$ has a probability distribution on $\mathcal{B}(\mathcal{P}(S))$.

Proof of Corollary 3.1. (1) Since every Polish space is Radon, $\nu \in \mathcal{P}(S)$ for each $\omega \in \Omega$; thus, $\nu$ is an $\mathcal{F}$-measurable Radon probability measure on $\mathcal{B}(S)$, so the first assertion follows from Proposition 3.4 .

(2) Since $X$ is a random element in $S$, from Proposition 3.3 the range space of $\theta \circ X$ is $\Sigma$. Furthermore, for $C \in \mathcal{B}(\Sigma)$,

$$
(\theta \nu)(C)=\nu\left(\theta^{-1} C\right)=\mathrm{P}^{\mathcal{F}}\left(X \in \theta^{-1} C\right)=\mathrm{P}^{\mathcal{F}}(\theta \circ X \in C) \quad \mathrm{P}_{\mathcal{F}} \text {-a.s. }
$$

so $\theta \nu$ is a cd of $\theta \circ X$ given $\mathcal{F}$. Also, $\theta \nu$ is an $\mathcal{F}$-measurable probability measure on $\mathcal{B}(\Sigma)$ since $(\theta \nu)(C)$ is $\mathcal{F}$-measurable in $\omega$ for every $C \in \mathcal{B}(\Sigma)$. By Proposition 2.1, for each $\omega \in \Omega$, the image measure $(\theta \nu)(\omega)=\hat{\nu}(\omega)$ is in $\mathcal{P}(\Sigma)$.

(3) Thus, $\hat{\nu}$ is an $\mathcal{F}$-measurable Radon probability measure on $\mathcal{B}(\Sigma)$. Since $\Sigma$ is compact Hausdorff by Proposition 3.3 , hence normal, Proposition 3.4 leads to the third assertion.

The proposition below gives the extended distribution of $\hat{\nu}$ in Corollary $3.1(2) \&(3)$. In the proof we shall use the following lemma.

Lemma 3.1. Let $\left(Z_{t}, \tau_{t}\right), t \in T$, be an indexed family of topological spaces, and $A$ a Borel set in $Z=\times_{t \in T} Z_{t}$ equipped with the product topology $\tau$. Assume that the relative topology $\tau_{A}=A \cap \tau$ has a countable base. Then

$$
A \cap \bigotimes_{t \in T} \mathcal{B}\left(Z_{t}\right)=A \cap \mathcal{B}\left(\underset{t \in T}{X} Z_{t}\right) .
$$


Proof. Let $\beta$ be a countable base for $\tau_{A}=A \cap \tau$. Every set $B \in \beta \subset$ $A \cap \tau$ is the union of some family $\Phi$ of finite intersections of cylinders like $A \cap\left(U_{t} \times \times_{s \in T-\{t\}} Z_{s}\right)$ with $U_{t} \in \tau_{t}$. Then $\Phi \subset A \cap \tau$. Since the topology $A \cap \tau$ has a countable base, by Lindelöf's theorem in Dunford and Schwartz $\left(1958\right.$, I.4.14, p. 12) there is a countable subfamily $\Phi_{0} \subset \Phi$ with $\bigcup \Phi_{0}=\bigcup \Phi$. Then $B=\bigcup \Phi_{0}$, so

$$
B \in \sigma\left(\left\{A \cap\left(U_{t} \times \underset{s \in T-\{t\}}{\times} Z_{s}\right), t \in T\right\}\right)=A \cap \bigotimes_{t \in T} \mathcal{B}\left(Z_{t}\right)
$$

hence, $\sigma(\beta) \subseteq A \cap \bigotimes_{t \in T} \mathcal{B}\left(Z_{t}\right)$.

Moreover, $\sigma(A \cap \tau)=\sigma(\beta)$ since $A \cap \tau$ has a countable base $\beta$. In addition,

$$
(\forall t \in T) \quad A \cap\left(U_{t} \times \underset{s \in T-\{t\}}{\times} Z_{s}\right) \in A \cap \tau,
$$

so $A \cap \bigotimes_{t \in T} \mathcal{B}\left(Z_{t}\right) \subseteq \sigma(A \cap \tau)=\sigma(\beta)$. Then $\sigma(\beta)=A \cap \bigotimes_{t \in T} \mathcal{B}\left(Z_{t}\right)$.

Since $\sigma(\beta)=\sigma(A \cap \tau)=A \cap \sigma(\tau)=A \cap \mathcal{B}\left(\mathrm{X}_{t \in T} Z_{t}\right)$, we get

$$
A \cap \bigotimes_{t \in T} \mathcal{B}\left(Z_{t}\right)=A \cap \mathcal{B}\left(\underset{t \in T}{X} Z_{t}\right)
$$

By assumption $A \in \mathcal{B}(Z)$, so $\mathcal{B}(A)=A \cap \mathcal{B}\left(\times_{t \in T} Z_{t}\right)$. Then the above equality means that every set of $\mathcal{B}(Z)$ contained in $A$ coincides with an intersection $C \cap A$ for some $C \in \bigotimes_{t \in T} \mathcal{B}\left(Z_{t}\right)$.

Proposition 3.5. Let $\hat{\nu}$, the Polish space $S$, the space $\Sigma$ and the set $\Psi$ be as in Corollary 3.1(3). For $\lambda \in \mathcal{P}(\Sigma)$, put $\hat{\zeta}(\lambda)=(\lambda(\psi), \psi \in \Psi)$ ), $\hat{\zeta}_{\psi}(\lambda)=\lambda(\psi)=\int_{\Sigma} \psi d \lambda, A_{\psi}=\hat{\zeta}_{\psi}[\mathcal{P}(\Sigma)] \subset \mathbb{R}_{\psi}$, and $\mathcal{B}_{\psi}=\mathcal{B}\left(\mathbb{R}_{\psi}\right)$; also, assume $\mathcal{P}(\Sigma)$ is always equipped with the narrow topology. With the map $\theta$ of Proposition 3.3, the map $h: \mu \mapsto \theta \mu$ is a homeomorphism from $\mathcal{P}(S)$ onto $\pi=h[\mathcal{P}(S)] \subset \mathcal{P}(\Sigma)$. Then the measure $\mu^{*}$ induced by the Kolmogorov distribution $\mu^{\prime}$ of the process $(\hat{\nu}(\psi), \psi \in \Psi)=\hat{\zeta}(\hat{\nu})$ on its range space $\hat{\zeta}(\pi)$ is the distribution of $\hat{\zeta}(\hat{\nu})$, and $\hat{\zeta}^{-1} \mu^{*}$, which is a Radon probability on $\mathcal{B}(\pi)$, is the distribution of the $\pi$-valued random point $\hat{\nu}$.

Proof. (1) Since $\Sigma$ in Proposition 3.3 is a compact Hausdorff space, $\mathcal{P}(\Sigma)$ endowed with the narrow topology is by Proposition 3.2 a compact Hausdorff space, hence normal. Then by Proposition $3.1 . \hat{\zeta}=\left(\hat{\zeta}_{\psi}, \psi \in \Psi\right)$ is a homeomorphism from $\mathcal{P}(\Sigma)$ into $A_{\Psi}=\mathrm{X}_{\psi \in \Psi} A_{\psi} \subset \bar{X}_{\psi \in \Psi} \mathbb{R}_{\psi}$, so, for each $\psi \in \Psi$, the map $\hat{\zeta}_{\psi}$ from $\mathcal{P}(\Sigma)$ onto $A_{\psi}=\hat{\zeta}_{\psi}[\mathcal{P}(\Sigma)]$ being continuous, $A_{\psi}$ is compact, hence closed in $\mathbb{R}_{\psi}$. Thus, $A_{\psi} \in \mathcal{B}_{\psi}$, and $\mathcal{B}\left(A_{\psi}\right)=A_{\psi} \cap \mathcal{B}_{\psi}$.

(2) In addition, for any finite subset $F$ of $\Psi,\left(\hat{\zeta}_{\psi}, \psi \in F\right): \mathcal{P}(\Sigma) \rightarrow$ $\times_{\psi \in F} \mathbb{R}_{\psi}$, so, for all $B \in \bigotimes_{\psi \in F} \mathcal{B}_{\psi}$, 


$$
\begin{aligned}
\left(\hat{\zeta}_{\psi}, \psi \in F\right)^{-1}(B) & =\left\{\lambda: \lambda \in \mathcal{P}(\Sigma),\left(\hat{\zeta}_{\psi}, \psi \in F\right)(\lambda) \in B\right\} \\
& =\left\{\lambda: \lambda \in \mathcal{P}(\Sigma), \hat{\zeta}(\lambda) \in B \times \underset{\psi \in \Psi-F}{\times} \mathbb{R}_{\psi}\right\}
\end{aligned}
$$

Moreover, $\left(B \times \times_{\psi \in \Psi-F} \mathbb{R}_{\psi}\right) \in \bigotimes_{\psi \in \Psi} \mathcal{B}_{\psi}$, so

$$
\left(\hat{\zeta}_{\psi}, \psi \in F\right)^{-1}\left(\bigotimes_{\psi \in F} \mathcal{B}_{\psi}\right) \subset \hat{\zeta}^{-1}\left(\bigotimes_{\psi \in \Psi} \mathcal{B}_{\psi}\right)
$$

(3) In Corollary 3.1 let $m$ be the distribution of $\hat{\nu}$ on the $\sigma$-field $\hat{\zeta}^{-1}\left(\bigotimes_{\psi \in \Psi} \mathcal{B}_{\psi}\right) \subset \mathcal{B}(\mathcal{P}(\Sigma))$. Since

$$
\left(\hat{\zeta}_{\psi}, \psi \in F\right)^{-1}\left(\bigotimes_{\psi \in F} \mathcal{B}\left(A_{\psi}\right)\right)=\left(\hat{\zeta}_{\psi}, \psi \in F\right)^{-1}\left(\bigotimes_{\psi \in F} \mathcal{B}_{\psi}\right)
$$

for $\mathrm{C} \in \bigotimes_{\psi \in F} \mathcal{B}\left(A_{\psi}\right)$ we have $\left(\hat{\zeta}_{\psi}, \psi \in F\right)^{-1}(\mathrm{C}) \in \hat{\zeta}^{-1}\left(\bigotimes_{\psi \in \Psi} \mathcal{B}_{\psi}\right)$, and

$$
\begin{aligned}
m\left[\left(\hat{\zeta}_{\psi}, \psi \in F\right)^{-1}(\mathrm{C})\right] & =\mathrm{P}\left[\hat{\nu} \in\left(\hat{\zeta}_{\psi}, \psi \in F\right)^{-1}(\mathrm{C})\right] \\
& =\mathrm{P}\left[\left(\hat{\zeta}_{\psi}, \psi \in F\right) \circ \hat{\nu} \in \mathrm{C}\right] .
\end{aligned}
$$

Thus, the image measure $\mu_{F}=\left(\hat{\zeta}_{\psi}, \psi \in F\right) m$ on the Borel $\sigma$-field $\mathcal{B}\left(\times_{\psi \in F} A_{\psi}\right)$ $=\bigotimes_{\psi \in F} \mathcal{B}\left(A_{\psi}\right)$ is the distribution of $\left(\hat{\zeta}_{\psi}, \psi \in F\right) \circ \hat{\nu}$.

(4) In addition, $\left(\hat{\zeta}_{\psi}, \psi \in F\right) \circ \hat{\nu}=\left(\hat{\zeta}_{\psi}(\hat{\nu}), \psi \in F\right), \hat{\zeta}_{\psi}(\hat{\nu})=\hat{\nu}(\psi)=$ $\int_{\Sigma} \psi d \hat{\nu}$, so $\mu_{F}$ is just a finite-dimensional probability distribution for the family $\hat{\zeta}(\hat{\nu})$ of random variables $\hat{\zeta}_{\psi}(\hat{\nu}), \psi \in \Psi$. Moreover, from the consistency theorem (cf. Loève, 1977, p. 94), the consistent system $\left\{\mu_{F}, F \subset \Psi\right\}$ uniquely determines a probability $\mu^{\prime}$ on the product $\sigma$-field $\bigotimes_{\psi \in \Psi}\left(\mathcal{B}_{\psi} \cap A_{\psi}\right)$ $=\bigotimes_{\psi \in \Psi} \mathcal{B}\left(A_{\psi}\right)$ in the product space $A_{\Psi}=\times_{\psi \in \Psi} A_{\psi}$; also, with $\pi_{F}$ being the canonical projection from $A_{\Psi}$ onto $\times_{\psi \in F} A_{\psi}$, the distribution $\mu^{\prime}$ is determined by the condition

$$
(\forall \text { F finite } \subset \Psi) \quad \mu^{\prime}\left(\pi_{\mathrm{F}}^{-1} \mathrm{~B}_{\mathrm{F}}\right)=\mu_{\mathrm{F}}\left(\mathrm{B}_{\mathrm{F}}\right), \quad \mathrm{B}_{\mathrm{F}} \in \bigotimes_{\psi \in \mathrm{F}} \mathcal{B}\left(\mathrm{A}_{\psi}\right) .
$$

(5) The map $\theta$ in Proposition 3.3 is a homeomorphism from $S$ onto $\theta(S) \subset \Sigma$. From Proposition 2.3, the map $h: \mu \mapsto \theta \mu$ is a homeomorphism from $\mathcal{P}(S)$ onto $\pi=h[\mathcal{P}(S)] \subset \mathcal{P}(\Sigma)$.

In Corollary $3.1(2), \theta \nu$ has been denoted by $\hat{\nu}$; moreover, the range space of $\nu$ is the Polish space $\mathcal{P}(S)$, so $\hat{\nu}$ has the image $\pi=h[\mathcal{P}(S)]$ for a range space. Moreover, according to Schwartz (1973, Definition 2, p. 94), as the continuous bijective image of the Polish space $\mathcal{P}(S), h[\mathcal{P}(S)]$ is a Luzin space contained in the Hausdorff space $\mathcal{P}(\Sigma)$; therefore, from Schwartz 1973 , Theorem 5, p. 101), $\pi=h[\mathcal{P}(S)]$ is a Borel set in $\mathcal{P}(\Sigma)$.

The Kolmogorov distribution $\mu^{\prime}$ has been defined on the $\sigma$-field $\bigotimes_{\psi \in \Psi} \mathcal{B}\left(A_{\psi}\right)$ in $\mathrm{X}_{\psi \in \Psi} A_{\psi}$. From Definition 2.2 , the induced measure $\mu_{\hat{\zeta}(\pi)}^{\prime}$ 
is defined on the $\sigma$-field $\hat{\zeta}(\pi) \cap \bigotimes_{\psi \in \Psi} \mathcal{B}\left(A_{\psi}\right)$. Since $\hat{\zeta}(\pi) \subset \hat{\zeta}(\mathcal{P}(\Sigma)) \subset$ $\mathrm{X}_{\psi \in \Psi} A_{\psi}$, we have $\hat{\zeta}(\pi) \in \hat{\zeta}(\pi) \cap \bigotimes_{\psi \in \Psi} \mathcal{B}\left(A_{\psi}\right)$ according to Definition 2.2. Moreover, $\hat{\zeta}(\hat{\nu}) \in \hat{\zeta}(\pi)$, and $\mu^{\prime}$ is the distribution of $\hat{\zeta}(\hat{\nu})$ on $\bigotimes_{\psi \in \Psi} \mathcal{B}\left(A_{\psi}\right)$, so, for the induced measure,

$$
\mu_{\hat{\zeta}(\pi)}^{\prime}(\hat{\zeta}(\pi))=\inf \left\{\mu^{\prime}(C): C \in \bigotimes_{\psi \in \Psi} \mathcal{B}\left(A_{\psi}\right), C \supset \hat{\zeta}(\pi)\right\}=1 .
$$

(6) Hence, $\mu_{\hat{\zeta}(\pi)}^{\prime}$ is a probability measure on $\hat{\zeta}(\pi) \cap \bigotimes_{\psi \in \Psi} \mathcal{B}\left(A_{\psi}\right)$. Since $\hat{\zeta}$ is a homeomorphism from $\mathcal{P}(\Sigma)$ into $A_{\Psi}=\times_{\psi \in \Psi} A_{\psi}$, and $\pi \in \mathcal{B}(\mathcal{P}(\Sigma)), \hat{\zeta}$ is a homeomorphism from $\pi$ equipped with the relative narrow topology onto $\hat{\zeta}(\pi)$ equipped with the relative product topology; thus, $\hat{\zeta}(\pi) \in \mathcal{B}\left(\mathrm{X}_{\psi \in \Psi} A_{\psi}\right)$. In addition, $\pi=h(\mathcal{P}(S))$ is homeomorphic to the Polish space $\mathcal{P}(S)$, hence $\pi$ is also a Polish space, and so is $\hat{\zeta}(\pi)$ equipped with the relative product topology. Let $\tau$ be the product topology of $\mathrm{X}_{\psi \in \Psi} A_{\psi}$; since $\hat{\zeta}(\pi)$ is a Polish space, the relative topology $\hat{\zeta}(\pi) \cap \tau$ has a countable base. By Lemma 3.1 .

$$
\hat{\zeta}(\pi) \cap \bigotimes_{\psi \in \Psi} \mathcal{B}\left(A_{\psi}\right)=\hat{\zeta}(\pi) \cap \mathcal{B}\left(\underset{\psi \in \Psi}{X} A_{\psi}\right)=\hat{\zeta}(\pi) \cap \mathcal{B}\left(A_{\Psi}\right) .
$$

Therefore, $\mu_{\hat{\zeta}(\pi)}^{\prime}$ is a probability measure on the $\sigma$-field $\hat{\zeta}(\pi) \cap \mathcal{B}\left(A_{\Psi}\right)=$ $\mathcal{B}(\hat{\zeta}(\pi))$.

(7) Now, define a probability measure $\mu^{*}$ on $\hat{\zeta}(\pi) \cap \mathcal{B}\left(A_{\Psi}\right)=\mathcal{B}(\hat{\zeta}(\pi))$ by $\mu^{*}=\mu_{\hat{\zeta}(\pi)}^{\prime}$. Since $\hat{\zeta}(\pi)$ is a Polish space, the Borel measure $\mu^{*}$ on $\mathcal{B}(\hat{\zeta}(\pi))$ is Radon.

(8) $\pi$ is the range space for $\hat{\nu}$, so $\hat{\zeta}(\pi)$ is the range space for $\hat{\zeta}(\hat{\nu})$. The Kolmogorov distribution $\mu^{\prime}$ is the distribution of $\hat{\zeta}(\hat{\nu})$ on the $\sigma$-field $\bigotimes_{\psi \in \Psi} \mathcal{B}\left(A_{\psi}\right)$. Therefore, with $C^{\prime} \in \bigotimes_{\psi \in \Psi} \mathcal{B}\left(A_{\psi}\right)$,

$$
P\left[\hat{\zeta}(\hat{\nu}) \in \hat{\zeta}(\pi) \cap C^{\prime}\right]=P\left[\hat{\zeta}(\hat{\nu}) \in C^{\prime}\right]=\mu^{\prime}\left(C^{\prime}\right) .
$$

Further, from Definition 2.2 .

$$
\begin{aligned}
\mu_{\hat{\zeta}(\pi)}^{\prime}\left[\hat{\zeta}(\pi) \cap C^{\prime}\right] & =\inf \left\{\mu^{\prime}(C): C \in \bigotimes_{\psi \in \Psi} \mathcal{B}\left(A_{\psi}\right), C \supset \hat{\zeta}(\pi) \cap C^{\prime}\right\} \\
& =\inf \left\{\mu^{\prime}(C): C \in \bigotimes_{\psi \in \Psi} \mathcal{B}\left(A_{\psi}\right), \hat{\zeta}(\pi) \cap C \supseteq \hat{\zeta}(\pi) \cap C^{\prime}\right\} \\
& =\inf \left\{\mu^{\prime}(C): C \in \bigotimes_{\psi \in \Psi} \mathcal{B}\left(A_{\psi}\right), \hat{\zeta}(\pi) \cap C=\hat{\zeta}(\pi) \cap C^{\prime}\right\} .
\end{aligned}
$$

Since, for $C, C^{\prime} \in \bigotimes_{\psi \in \Psi} \mathcal{B}\left(A_{\psi}\right)$, if $\hat{\zeta}(\pi) \cap C=\hat{\zeta}(\pi) \cap C^{\prime}$ then $\hat{\zeta}(\pi) \cap\left(C \triangle C^{\prime}\right)$ $=\emptyset$, so $\mu^{\prime}\left(C \triangle C^{\prime}\right)=0$, i.e. $\mu^{\prime}(C)=\mu^{\prime}\left(C^{\prime}\right)$. It follows that

$$
\mu_{\hat{\zeta}(\pi)}^{\prime}\left[\hat{\zeta}(\pi) \cap C^{\prime}\right]=\mu^{\prime}\left(C^{\prime}\right)=P\left[\hat{\zeta}(\hat{\nu}) \in \hat{\zeta}(\pi) \cap C^{\prime}\right] .
$$


Thus, $\mu^{*}=\mu_{\hat{\zeta}(\pi)}^{\prime}$ is the distribution of $\hat{\zeta}(\hat{\nu})$ on $\hat{\zeta}(\pi) \cap \bigotimes_{\psi \in \Psi} \mathcal{B}\left(A_{\psi}\right)=\hat{\zeta}(\pi) \cap$ $\mathcal{B}\left(A_{\Psi}\right)=\mathcal{B}(\hat{\zeta}(\pi))$. Hence, $\hat{\zeta}^{-1}$ being a homeomorphism from $\hat{\zeta}(\pi)$ onto $\pi$, $\hat{\zeta}^{-1} \mu^{*}$ is the distribution of $\hat{\nu}$ on $\mathcal{B}(\pi)$, and $\hat{\zeta}^{-1} \mu^{*}$ is a Radon probability on $\mathcal{B}(\pi)$ by Proposition 2.1.

Finally, we are ready to prove the following, attaining our aim.

Theorem 3.1. Let $S$ be a Polish space, $X:(\Omega, \mathcal{A}) \rightarrow(S, \mathcal{B}(S))$ a random element in $S$, and $\mathcal{F}$ any sub- $\sigma$-field of $\mathcal{A}$. Let $\nu$ be a conditional distribution of $X$ given $\mathcal{F}$. Then $\nu \in \mathcal{P}(S)$ for all $\omega \in \Omega$, and $\nu$ has a distribution which is a Radon probability measure on the Borel $\sigma$-field $\mathcal{B}(\mathcal{P}(S))$ of the space $\mathcal{P}(S)$ supplied with the narrow topology.

Proof. Since any Polish space is Radon, $\nu \in \mathcal{P}(S)$ for all $\omega \in \Omega$.

In the proof of Proposition 3.5, $h: \mu \mapsto \theta \mu$ is a homeomorphism from $\mathcal{P}(S)$ onto $\pi=h[\mathcal{P}(S)]$, and $h^{-1}$ is one from $\pi$ onto $\mathcal{P}(S)$, so Borel sets $C \in \mathcal{B}(\pi)$ bijectively correspond to Borel sets $B \in \mathcal{B}(\mathcal{P}(S))$; thus, for $\hat{\nu}=\theta \nu$, we have $\hat{\nu} \in C \Leftrightarrow \nu \in B$, and $C=h(B)$. Also, by denoting $\hat{\eta}_{\pi}=\hat{\zeta}^{-1} \mu^{*}$, from Proposition 2.1 the image measure $h^{-1} \hat{\eta}_{\pi}$ of the Radon probability $\hat{\zeta}^{-1} \mu^{*}$ in Proposition 3.5 is a Radon probability on $\mathcal{P}(S)$. Furthermore, since $\hat{\zeta}^{-1} \mu^{*}$ is the distribution of $\hat{\nu}$ on $\mathcal{B}(\pi)$, we have

$$
\hat{\eta}_{\pi}(C)=P(\hat{\nu} \in C)=P(\nu \in B) .
$$

In addition,

$$
\hat{\eta}_{\pi}(C)=\hat{\eta}_{\pi}(h(B))=\left(h^{-1} \hat{\eta}_{\pi}\right)(B),
$$

so $h^{-1} \hat{\eta}_{\pi}$ is the distribution of $\nu$ on $\mathcal{B}(\mathcal{P}(S))$, and $h^{-1} \hat{\eta}_{\pi}$ is a Radon measure since the space $\mathcal{P}(S)$ supplied with the narrow topology is Polish by Proposition 2.2 , hence a Radon space.

A random probability measure on a Polish space $(S, \mathcal{B}(S))$ will be called a Radon random probability measure if it has a determined distribution on $\mathcal{B}(\mathcal{P}(S))$. Thus, Theorem 3.1 asserts that an infinite, exchangeable sequence in a Polish space is directed (cf. Kallenberg, 2005, Proposition 1.4(i), p. 28) by a Radon random probability measure. In other words, we have the following

CONCLUSION 3.1. The prior distribution of the a.s. unique random measure underlying an infinite, exchangeable sequence of observations from a Polish space $S$ is a Radon probability measure on the $\sigma$-field $\mathcal{B}(\mathcal{P}(S))$ in the Polish space $\mathcal{P}(S)$ equipped with the narrow topology.

Acknowledgements. The author would like to thank a reviewer for invaluable comments which helped to improve the presentation of the paper. 


\section{References}

N. Dunford and J. T. Schwartz (1958), Linear Operators. I. General Theory, Interscience Publ., London.

T. S. Ferguson (1973), A Bayesian analysis of some nonparametric problems, Ann. Statist. 1, 209-230.

B. de Finetti (1931), Funzione caratteristica di un fenomeno aleatorio, Rend. Lincei Mat. Appl. (6) 4, 251-299.

O. Kallenberg (2002), Foundations of Modern Probability, 2nd ed., Springer, New York.

O. Kallenberg (2005), Probabilistic Symmetries and Invariance Principles, Springer, New York.

O. Kallenberg (2017), Random Measures, Theory and Applications, Springer, Cham.

M. Loève (1977), Probability Theory, I, 4th ed., Springer, New York.

J. C. Oxtoby and S. M. Ulam (1939), On the existence of a measure invariant under a transformation, Ann. of Math. (2) 40, 560-566.

L. C. G. Rogers and D. Williams (1994), Diffusions, Markov Processes, and Martingales. I. Foundations, 2nd ed., Wiley, Chichester.

L. Schwartz (1973), Radon Measures on Arbitrary Topological Spaces and Cylindrical Measures, Tata Inst. Fund. Res., Bombay, Oxford Univ. Press, London.

Nguyen Bac-Van

Department of Statistics

VNUHCM-University of Science

227 Nguyen-Van-Cu Q5

Ho Chi Minh City, Vietnam

E-mail: nbvan@hcmus.edu.vn 